\title{
Short-Term Effect of Laser Acupuncture on Lower Back Pain: A Randomized, Placebo-Controlled, Double-Blind Trial
}

\author{
Jae-Young Shin, ${ }^{1}$ Boncho Ku, ${ }^{2}$ Jaeuk U. Kim, ${ }^{2}$ Yu Jung Lee, ${ }^{3}$ Jae Hui Kang, ${ }^{4}$ Hyun Heo, ${ }^{5}$ \\ Hyo-Joon Choi, ${ }^{5}$ and Jun-Hwan Lee ${ }^{1}$ \\ ${ }^{1}$ Clinical Research Division, Korea Institute of Oriental Medicine, Daejeon 34054, Republic of Korea \\ ${ }^{2}$ KM Fundamental Research Division, Korea Institute of Oriental Medicine, Daejeon 34054, Republic of Korea \\ ${ }^{3}$ Technology Licensing \& Commercialization Team, Korea Institute of Oriental Medicine, Daejeon 34054, Republic of Korea \\ ${ }^{4}$ Department of Acupuncture \& Moxibustion Medicine, College of Oriental Medicine, Daejeon University, \\ Daejeon 34520, Republic of Korea \\ ${ }^{5}$ Solco Biomedical Co., Ltd., Pyeongtaek, Gyeonggi 17704, Republic of Korea \\ Correspondence should be addressed to Jun-Hwan Lee; omdjun@kiom.re.kr
}

Received 29 May 2015; Revised 20 July 2015; Accepted 2 August 2015

Academic Editor: Baiyun Zeng

Copyright (C) 2015 Jae-Young Shin et al. This is an open access article distributed under the Creative Commons Attribution License, which permits unrestricted use, distribution, and reproduction in any medium, provided the original work is properly cited.

\begin{abstract}
Purpose. This trial was performed to investigate the efficacy of laser acupuncture for the alleviation of lower back pain. Methods. This was a randomized, placebo-controlled, double-blind trial. Fifty-six participants were randomly assigned to either the laser acupuncture group $(n=28)$ or the sham laser acupuncture group $(n=28)$. Participants in both groups received three treatment sessions over the course of one week. Thirteen acupuncture points were selected. The visual analogue scale for pain, pressure pain threshold, Patient Global Impression of Change, and Euro-Quality-of-Life Five Dimensions questionnaire (Korean version) were used to evaluate the effect of laser acupuncture treatment on lower back pain. Results. There were no significant differences in any outcome between the two groups, although the participants in both groups showed a significant improvement in each assessed parameter relative to the baseline values. Conclusion. Although there was no significant difference in outcomes between the two groups, the results suggest that laser acupuncture can provide effective pain alleviation and can be considered an option for relief from lower back pain. Further studies using long-term intervention, a larger sample size, and rigorous methodology are required to clarify the effect of laser acupuncture on lower back pain.
\end{abstract}

\section{Introduction}

Lower back pain (LBP) is one of the most common pathological conditions, affecting $80-85 \%$ of individuals at least once during their lifetime and exhibiting a yearly prevalence rate of approximately $15-45 \%$. Aggravation of this pain, which is a frequent occurrence, leads to serious social and economic burdens for patients and limits their activity [1].

The "obstruction of qi and blood in the meridian" is one of the major causes of LBP [2]. Acupuncture stimulates specific points to relieve obstruction in the channels, rectify qi, and stimulate blood circulation [3]. Accordingly, it is commonly used to alleviate LBP and is an effective treatment for this condition. However, patients may be reluctant to accept this treatment because it requires frequent visits to the doctor. In addition, patients fear the possibility of pain and inflammation related to the use of needles. For pain relief, patients may instead use ointments or plasters, which are convenient but do not provide any obvious or long-lasting effects.

Therefore, it is necessary to develop new equipment as an effective replacement for conventional acupuncture equipment. The technology used to stimulate acupuncture points has advanced, shifting from stone to metal, and has now advanced to laser acupuncture [4]. The use of laser light as an alternative to stimulate acupuncture points has been promoted for almost three decades [5]. Generally referred to as laser acupuncture, it has been clinically applied since 
the 1970s and was made possible by the application of a lowintensity laser [4]. Previous studies have shown that laser acupuncture can stimulate acupuncture points for neck pain treatment [6]. Furthermore, low level laser therapy (LLLT) on wound areas as well as on acupuncture points, as a noninvasive, pain-free method with minor side effects, has been considered as a possible treatment option for the diabetic foot syndrome [7]. In addition, several studies have reported that laser acupuncture is effective for the treatment of many diseases, such as chronic tension headache, fibromyalgia, and chronic neck pain [8-10].

The aim of this study was to develop a convenient and safe laser acupuncture technique with satisfactory efficacy for the treatment of lower back pain and to record its therapeutic effects.

\section{Methods}

2.1. Study Design. We conducted a randomized, placebocontrolled, double-blind parallel study to compare the effect of sham laser acupuncture with laser acupuncture. The study was carried out at the Department of Acupuncture \& Moxibustion, Cheonan Korean Medicine Hospital of Daejeon University, Republic of Korea.

Fifty-six participants with LBP were recruited through advertisement. Following radiographic diagnosis, each participant received an explanation of the study procedure and signed the consent form. Vital signs and relevant histories were recorded. The participants were randomly assigned to either the laser acupuncture group $(n=28)$ or the sham laser acupuncture group $(n=28)$, and they received laser or sham laser stimuli at five bilateral acupuncture points and three midline acupuncture points for three minutes. Every participant received three treatment sessions over the course of one week. The treatment interval ranged from two to four days. Interviews and clinical examinations were conducted within one week after the last treatment session. The study design is depicted in Figure 1.

2.2. Randomized Allocation and Blinding. The participant randomization list was compiled by an external investigator and was not divulged to other investigators or the participants. The external investigator prepared a series of sealed, sequentially numbered envelopes containing the treatment assignments. When a participant fulfilled the inclusion criteria, the study investigator opened the lowest numbered envelope to reveal the group allocation. Blinding was achieved for both the investigator and the participant, without revealing either the laser acupuncture or the placebo acupuncture treatment.

2.3. Participants. The inclusion criteria for the men and women in the study were as follows: (1) age range of 20 to 75 years; (2) minimum pain intensity of $30 \mathrm{~mm}$ on the visual analogue scale (VAS) for pain, which ranges from 0 to $100 \mathrm{~mm}$; (3) a diagnosis of lower back pain; (4) the ability to participate voluntarily, clearly understand the purpose and characteristics of the clinical trial, and sign an informed

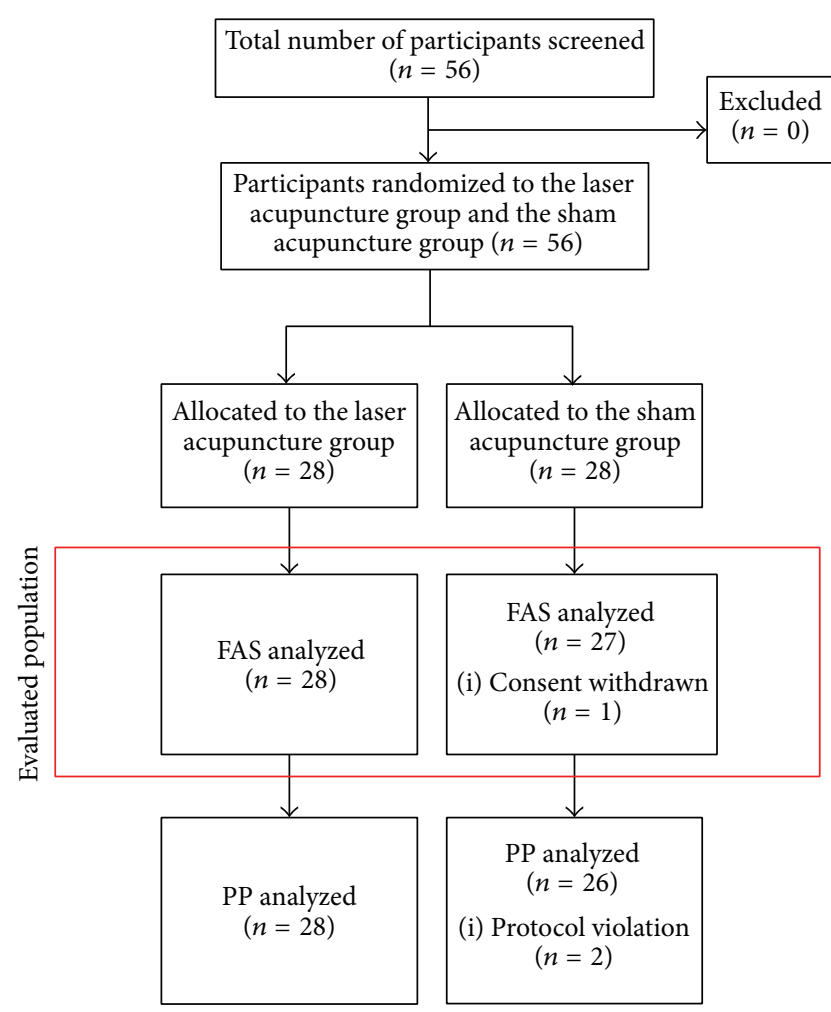

FIGURE 1: Study flowchart of the laser acupuncture randomized, placebo-controlled, double-blind trial for lower back pain. FAS: full analysis set; PP: per protocol.

consent form. Participants were excluded from the study if one or more of the following criteria were fulfilled: (1) the use of devices such as pacemakers and hearing aids that could be affected by electromagnetic fields; (2) presence of severe pain that precluded participation in a clinical trial; (3) diagnoses of fractures, severe disc herniation, or spinal tumors, which require immediate examination and treatment; (4) use of medication, such as corticosteroids, anticonvulsants, and anti-inflammatory drugs that could affect the outcome of the trial; (5) pregnancy; (6) prior history of adverse effects, to physical stimulation therapy; (7) significant physical or mental deficiencies preventing a clear understanding of the clinical trial procedure; (8) participation in other clinical trials within the previous month.

2.4. Ethics Statement. The study protocol was approved by the Ethics Committee of Cheonan Korean Medicine Hospital of Daejeon University, Korea. Written informed consent was obtained from all participants in accordance with the Declaration of Helsinki.

2.5. Interventions. All interventions were conducted at the Department of Acupuncture \& Moxibustion, Cheonan Korean Medicine Hospital of Daejeon University.

Laser acupuncture (a combination of medical laser irradiation equipment and cupping) was performed with an infrared 4-channel laser of low intensity (Solco-LF100, 


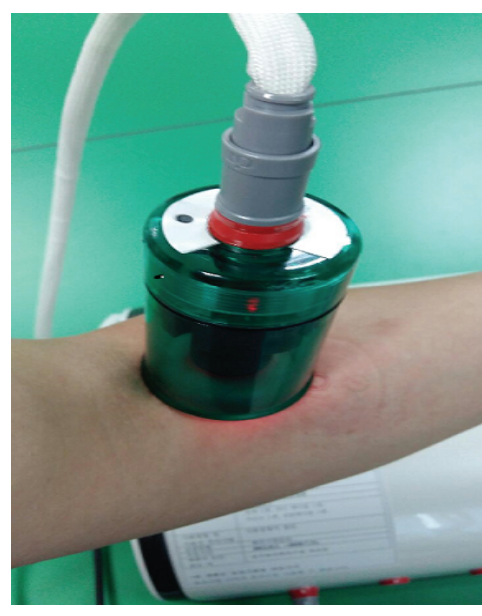

FIGURE 2: The intervention was performed with a cup-shaped laser acupuncture device.

Solco Biomedical Co., Ltd., Pyeongtaek, Korea) (Figure 2). The maximum output power was $53 \mathrm{~mW}$ with a laser light wavelength of $660 \mathrm{~nm}$ (pulse-type wave). The irradiated area of the skin was $2 \times 2 \mathrm{~mm}^{2}$, frequency was $200 \mathrm{~Hz}$, duty ratio was $50 \%$, pressure was $15 \mathrm{kPa}$, and output power was $50 \mathrm{~mW}$. Each treatment session lasted three minutes. This laser device is also equipped by the manufacturer with visual (red, light-emitting diode) and acoustic signals. Treatment was performed in sequence at 13 commonly used acupuncture points: unilateral GV3, GV4, and GV5 and bilateral BL23, BL24, BL25, BL40, and GB30 (Figure 3). The distance between the skin and laser was $5 \mathrm{~mm}$. The acupuncture points were localized without tactile irritation.

The sham laser acupuncture group underwent the same procedure as the laser acupuncture group, but the laser was not turned on. The acoustic functions of the equipment were preserved to ensure blinding. The sham laser acupuncture was performed according to the medical laser irradiation equipment treatment protocol.

We recorded the visual analogue scale (VAS) for pain and the pressure pain threshold (PPT) during visits 1-4 and measured the patient global impression of change (PGIC) and the Korean version of Euro-Quality-of-Life Five Dimensions (EQ-5D) at visits 1 and 4.

During the clinical trial period, other treatments for spinal sprain (except as prescribed in the protocol) were prohibited. The study institution offered a kinesiology guidebook to all participants to use for daily exercise therapy for LBP.

\subsection{Outcome Measures}

2.6.1. Visual Analogue Scale (VAS) for Pain. The $100 \mathrm{~mm}$ VAS is an instrument for the self-assessment of pain. Each participant marked the level of pain, and the VAS score was determined by measuring the distance between the left-hand end of the line and the marked point. We recorded VAS scores during visits $1-4$. The scores reported at visit 1 were used for screening purposes and as the baseline measurements.

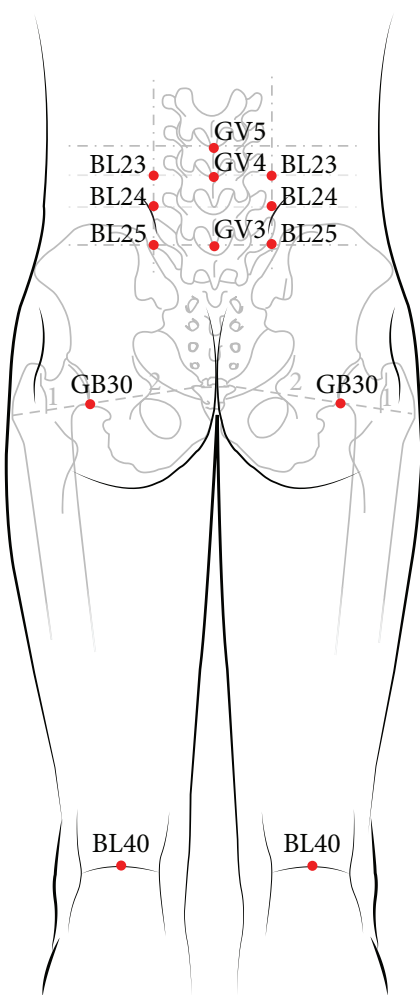

Figure 3: GV3, GV4, and GV5 and both sides of BL23, BL24, BL25, BL40, and GB30 (13 acupuncture points).

2.6.2. Pressure Pain Threshold (PPT). The PPT is defined as the minimum applied force that induces pain. The investigator placed a pressure algometer on BL25, instructed participants to indicate the first sensation of slight pain either vocally or by raising their hands, and then measured the pressure $\left(\mathrm{kg} / \mathrm{cm}^{2}\right)$. We recorded PPT scores during visits 14. The scores obtained at visit 1 were used as the baseline measurements.

2.6.3. Patient Global Impression of Change (PGIC). The PGIC evaluates the changes (if any) in activity limitations, symptoms, emotions, and overall quality of life which are related to painful conditions, as described by the participants from the beginning of the treatment. Each dimension has seven levels: (1) no change, or the condition is worse; (2) almost the same, with little change; (3) better, but no noticeable change; (4) somewhat better, but the change has made no real difference; (5) moderately better, with a slight but noticeable change; (6) better, with a definite improvement that has made a real and worthwhile difference; (7) a great deal better, with a considerable improvement that has made a marked difference. Participants chose the level that matched the degree of change. We assessed the PGIC at visits 1 and 4 .

2.6.4. Euro-Quality-of-Life Five Dimensions (EQ-5D, Korean Version). The EQ-5D is a standardized measure of health status developed by the EuroQol Group in order to provide a simple, generic measure of health for clinical and 
TABLE 1: Baseline characteristics.

\begin{tabular}{|c|c|c|c|}
\hline \multirow[b]{2}{*}{ Characteristics } & \multicolumn{2}{|c|}{ Group } & \multirow[b]{2}{*}{$P$ value } \\
\hline & $\begin{array}{l}\text { The laser acupuncture group } \\
\qquad(n=28)\end{array}$ & $\begin{array}{l}\text { The sham laser acupuncture group } \\
\qquad(n=27)\end{array}$ & \\
\hline $\mathrm{Age}^{\dagger}$ & $46.32(10.2)[21,47.5,65]$ & $46.48(11.68)[23,47,68]$ & 0.957 \\
\hline \multicolumn{4}{|l|}{ Sex $^{*}$} \\
\hline Female & $24\langle 85.7\rangle$ & $22\langle 81.5\rangle$ & 0.7287 \\
\hline Male & $4\langle 14.3\rangle$ & $5\langle 18.5\rangle$ & \\
\hline Duration of back pain (month) $)^{\dagger \dagger}$ & $13.78(19.58)[0.2,4.2,75.19]$ & $16.33(28.13)[0.2,6.18,130.98]$ & 0.8531 \\
\hline Compliance rate $(\%)^{\dagger \dagger}$ & $100(0)[100,100,100]$ & $97.53(12.83)[33.3,100,100]$ & 0.3261 \\
\hline Kinesitherapy $^{\dagger \dagger}$ & $2.75(0.44)[2,3,3]$ & $2.56(0.75)[0,3,3]$ & 0.4192 \\
\hline $\operatorname{BMI}\left(\mathrm{kg} / \mathrm{m}^{2}\right)^{\dagger}$ & $26.05(7.26)[16.4,25.2,59.19]$ & 23.39 (3.05) $[17.9,23.26,33.69]$ & 0.0844 \\
\hline Systolic blood pressure $(\mathrm{mmHg})^{\dagger}$ & $122.96(10.71)[97,124.5,143]$ & $115.15(11.48)[98,114,142]$ & 0.0117 \\
\hline Diastolic blood pressure $(\mathrm{mmHg})^{\dagger}$ & $74.75(10.55)[55,76,102]$ & $70.3(7.25)[59,71,89]$ & 0.0748 \\
\hline Pulse $(\mathrm{bpm})^{\dagger}$ & $73.71(10.3)[56,71.5,95]$ & $74.96(8.46)[61,74,90]$ & 0.6259 \\
\hline Body temperature $\left({ }^{\circ} \mathrm{C}\right)^{\dagger}$ & $36.57(0.36)[35.8,36.5,37.3]$ & $36.47(0.44)[35.4,36.5,37.4]$ & 0.3398 \\
\hline \multicolumn{4}{|l|}{ Baseline value } \\
\hline $\mathrm{VAS}^{\dagger}$ & $44.64(11.86)[30,40,75]$ & $47.78(10.95)[30,40,80]$ & 0.3135 \\
\hline PGIC & $4(0)[4,4,4]$ & $4(0)[4,4,4]$ & \\
\hline $\mathrm{PPT}^{\dagger}$ & $7.12(2.27)[3.1,7.2,11.92]$ & $6.56(1.86)[3.7,6.23,12.15]$ & 0.3231 \\
\hline $\mathrm{EQ}-5 \mathrm{D}^{\dagger}$ & $0.79(0.08)[0.6,0.8,0.9]$ & $0.75(0.1)[0.5,0.77,0.86]$ & 0.1056 \\
\hline
\end{tabular}

Values represent mean (SD) [min, median, max] for continuous variables and $N(\%)$ for categorical variables.

${ }^{\dagger}$ Independent two-sample $t$-test, ${ }^{\dagger \dagger}$ Wilcoxon's rank sum test, and ${ }^{*}$ Chi-squared test.

BMI: body mass index; VAS: visual analogue scale; PGIC: patient global impression of change; PPT: pressure pain threshold; EQ-5D: Euro-Quality-of-Life Five Dimensions.

economic appraisal. The EQ-5D consists of two parts: the EQ-5D descriptive system and the EQ visual analogue scale (EQ VAS). The EQ-5D descriptive system comprises the following five dimensions: mobility, self-care, usual activities, pain/discomfort, and anxiety/depression. Each dimension has three levels: no problems, some problems, and severe problems. Respondents were asked to indicate their health state by ticking (or placing a cross) in the box against the most appropriate statement in each of the five dimensions. We gave participants this questionnaire at visits 1 and 4 .

2.7. Statistical Analysis. The sample size was calculated using the conventional power analysis method. Twenty- eight participants were recruited in each group.

The software package $\mathrm{R}$ (version 3.0.1, " $\mathrm{R} \& \mathrm{R}$ ” of the Statistics Department of the University of Auckland, Auckland, New Zealand) was used for statistical analyses. Efficacy measurements were adjusted by the full analysis set. Missing values were input by using the last observation carried forward method.

The intergroup comparison of means (before and after the intervention) was processed by the analysis of covariance (ANCOVA), and the intragroup comparison of mean values (before and after the intervention) was analyzed by the paired $t$-test or the Wilcoxon signed rank sum test. The entire statistical analysis was a one-sided test. $P<0.05$ was considered statistically significant.

2.8. Adverse Events. The investigator recorded adverse events and unexpected responses to laser acupuncture treatment.
Adverse events were reported by the participants and evaluated by the investigator as mild, moderate, or severe according to the World Health Organization Draft Guidelines for Adverse Event Reporting [11] and Spilker's criteria [12].

\section{Results}

3.1. Demographic Characteristics and Baseline Values. In total, 56 participants with LBP were recruited for this study. All 28 participants assigned to the laser acupuncture group completed the study protocol. Of the 28 participants assigned to the sham laser acupuncture group, two failed to complete the protocol, as shown in Figure 1.

Participants in the two groups did not differ significantly in age, sex, duration, kinesitherapy, body mass index, diastolic blood pressure, pulse, or body temperature. However, systolic blood pressure differed significantly between participants in the groups (Table 1).

After collecting the data, we analyzed the results of the two participant groups. Baseline values (for VAS, PPT, PGIC, and EQ-5D) did not differ significantly between the two groups, as listed in Table 1.

3.2. Primary Outcome: Visual Analogue Scale (VAS) for Pain. The VAS scores changed significantly between visit 1 (baseline) and visit $4(P<0.001)$ and also between visit 1 (baseline) and visit $4(P<0.001)$ in both groups. However, the intergroup comparison of mean values (before and after 


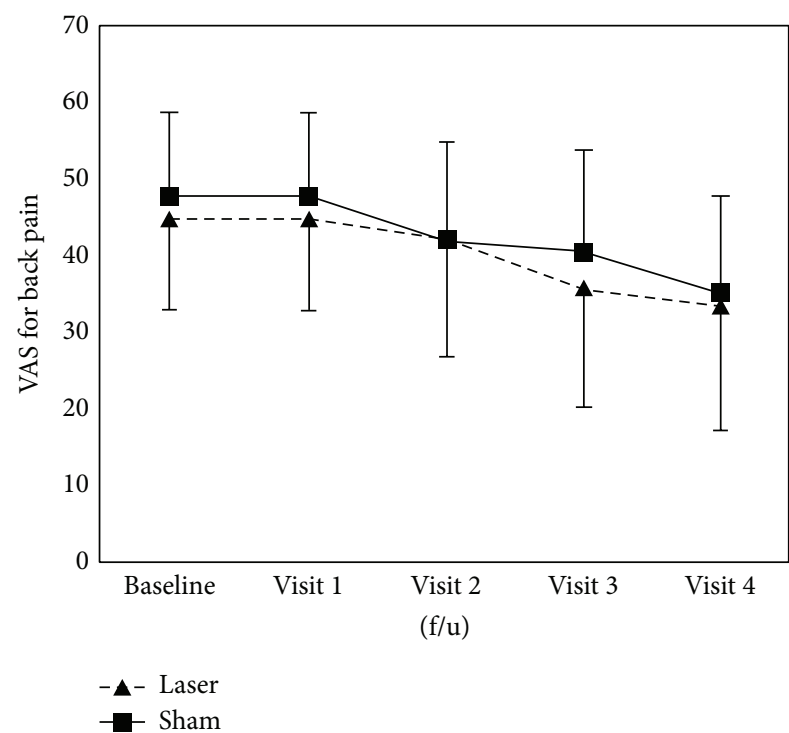

FIGURE 4: Change in visual analogue scale (VAS) for pain scores after intervention in both groups.

the intervention) did not reveal a significant difference in the VAS scores of the two groups (Figure 4 and Table 2).

3.3. Secondary Outcomes. Data from the pain questionnaires are presented in Table 2 . The results of secondary outcome measures were similar to those of the primary outcome measure. The PPT scores assessed at each time point compared with visit 1 (baseline) were significantly reduced in both groups $(P<0.01)$. The changes in PGIC between visit 1 (baseline) and visit 4 differed within both groups $(P<$ 0.001). The EQ-5D questionnaire outcomes of both groups improved throughout the trial (laser acupuncture group, $P<$ 0.05 ; sham laser acupuncture group, $P<0.01$ ). The results reveal that the PPT, PGIC, and EQ-5D scores changed in both groups. However, we found no significant differences between the two groups in the secondary outcome measures (Figures 5-7, Table 2).

3.4. Adverse Events. There were no indications of adverse effects (AE), such as erythema, itching, or blistering. No serious adverse events (SAE) were reported.

\section{Discussion}

4.1. Summary of Main Findings. The purpose of this study was to compare the efficacy of laser acupuncture with that of sham laser acupuncture for the treatment of LBP. Fifty-six participants with LBP were recruited and randomly assigned to either the laser acupuncture group or the sham laser acupuncture group. Participants in the two groups showed no significant differences in demographics; the significant difference in systolic blood pressure between the groups could have been incidental.

The intervention in the treatment group was performed with a cup-shaped laser acupuncture device (Figure 2);

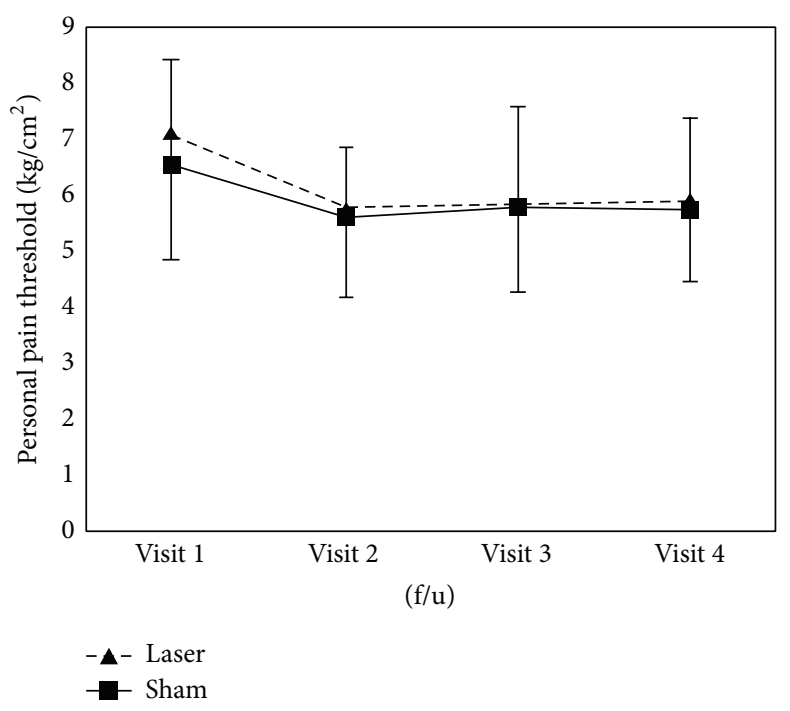

FIGURE 5: Change in pressure pain threshold (PPT) scores after intervention in both groups.

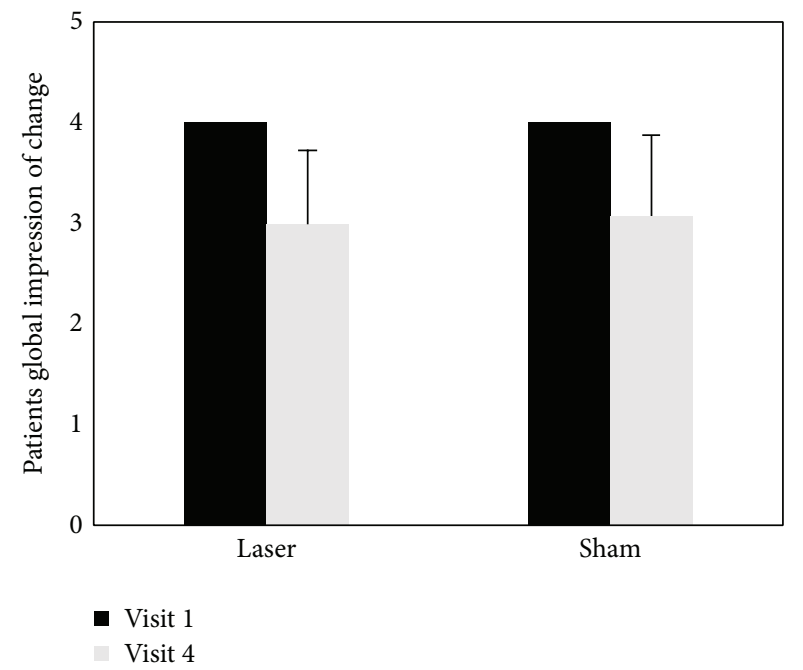

FIGURE 6: Change in patient global impression of change (PGIC) scores after intervention in both groups.

the same device with the laser turned off was used in control group. To treat LBP, acupuncture expert predefined a group of acupuncture points on 3 types of meridian (gallbladder meridian, GB; bladder meridian, BL; Governor Vessel, GV) according to the pain location. The Oriental Medicine is based on the theory that "There is stoppage, there is pain," which means that pain is caused by the obstruction of qi and blood in the meridian. The traditional acupuncturemoxibustion theory states the following: "Rescue the waist and back from pain by the Weizhong (BL40)." On the basis of these theories, unilateral GV3, GV4, and GV5 and bilateral BL23, BL24, BL25, BL40, and GB30 are often used for pain relief of LBP (Figure 3). In this study, we recorded the VAS, PPT, PGIC, and EQ-5D of all participants before and after intervention. Although the scores improved in both groups, 


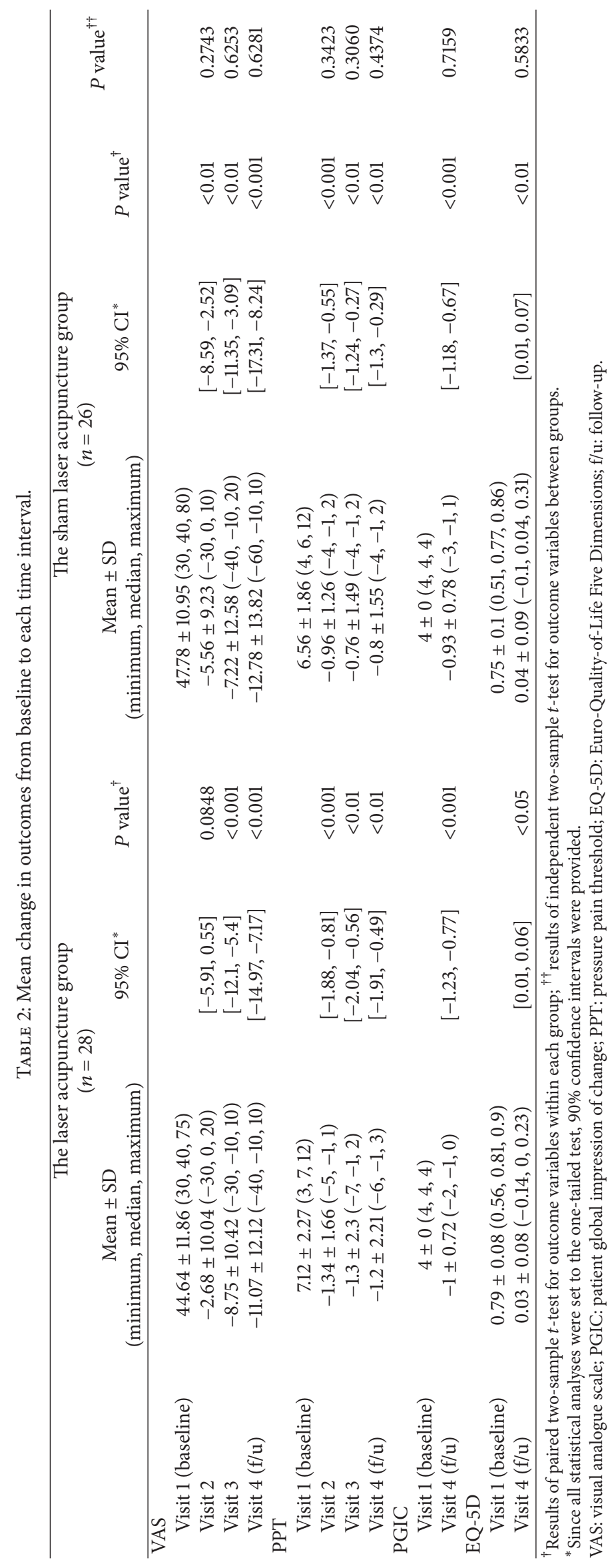




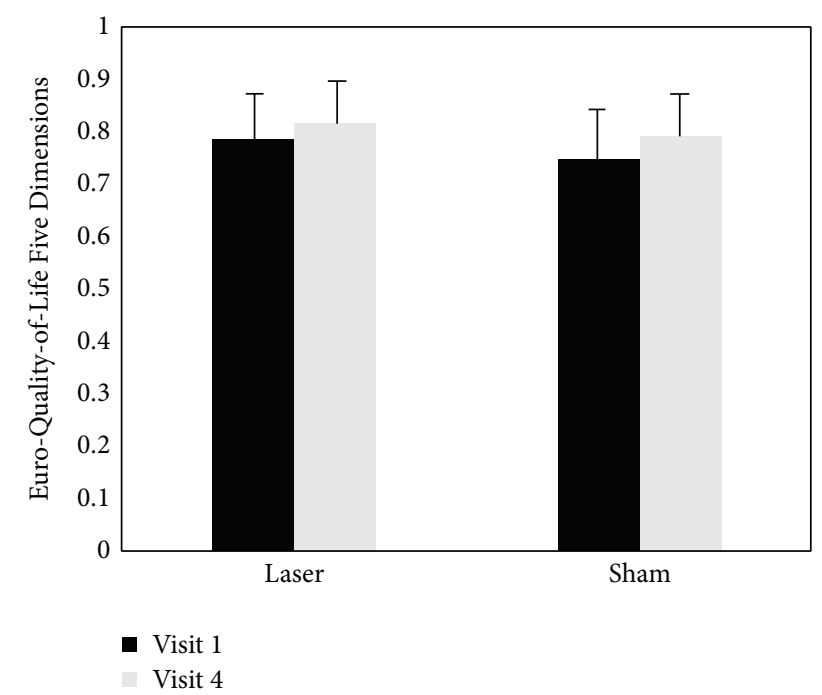

FIGURE 7: Change in Euro-Quality-of-Life Five Dimensions (EQ$5 \mathrm{D}$, Korean Version) scores after intervention in both groups.

the improvement in symptoms was not significantly different between the laser acupuncture group and the sham laser acupuncture group. It is possible that suction of the cupping device stimulated the lesion locations. Cupping therapy by partial vacuum has been found to improve the local blood and lymphatic circulation, increase the temperature of the local skin, and relieve painful muscle tension [13]. To hold the device to the skin without causing physiologic or therapeutic effects, we designed the suction pressure within the cupping glass $(15 \mathrm{kPa}=112.509 \mathrm{mmHg})$ to be weaker than that of normal cupping therapy $(600 \mathrm{mmHg})[14]$. However, we cannot exclude the possibility that cupping suction could trigger an analgesic effect. The development of a study control that is convenient and can also minimize physiologic effects will be important for future randomized controlled clinical trials for laser acupuncture.

4.2. Study Limitations. The participants in our study received only three treatments, because we sought to observe the short-term effects of laser acupuncture. According to one reference, approximately 10 treatment sessions are appropriate for acupuncture treatment [15]. In a systematic review of 16 laser acupuncture trials, the average number of treatment sessions was 9.6 [16]. Future studies on this topic are necessary to evaluate the optimum number of treatments.

The deqi, which is associated with the acupuncture effect, is one of the differences between acupuncture and placebo acupuncture [17]. Beissner and Marzolff reported that more than $80 \%$ of the participants experienced deqi after treatment by laser acupuncture [18]. Therefore, we suggest that further studies investigate the presence of deqi using questionnaire surveys to evaluate whether laser acupuncture could serve as an alternative to metal acupuncture and whether sham laser acupuncture is suitable for the control group.
Acupuncture textbooks suggest that the optimal depth of acupuncture stimulation in clinical treatment is 10.3$90.3 \mathrm{~mm}$ [15]. However, the laser wavelength in this study was fixed at $600 \mathrm{~nm}$ and penetrated approximately $5.5 \mathrm{~mm}$ into the acupuncture points, and it was impossible to change the depth of laser acupuncture stimulation. Because metal acupuncture needles are available in a variety of lengths and gauges, development of laser acupuncture devices with an adjustable wavelength and intensity of light should be considered.

\section{Conclusion}

This study compares laser acupuncture with sham laser acupuncture for the treatment of LBP. Substantial adverse events did not occur in the intervention period. Our results show that laser acupuncture is a relatively safe treatment that helps improve pain and the quality of life of patients with LBP. We recommend that future studies use longterm intervention, larger participant numbers, and rigorous methodology to determine the effect of laser acupuncture on LBP.

\section{Conflict of Interests}

The authors declare that they have no competing interests.

\section{Authors' Contribution}

Jaeuk U. Kim conceived the idea for the study and led protocol development. Boncho Ku, Yu Jung Lee, Jae Hui Kang, Hyun Heo, Hyo-Joon Choi, and Jun-Hwan Lee assisted with the study concept, study design, clinical interpretation, and paper drafting and finalization. Jun-Hwan Lee provided overall supervision as the primary mentor and led the team in paper preparation. Jae-Young Shin wrote the paper and approved the final version for publication. All authors commented on drafts of the paper and approved its final version. Jae-Young Shin contributed to this work.

\section{Acknowledgments}

This study was supported by the Korea Institute of Oriental Medicine (Grants nos. K15121 and K15020) and the Technology Innovation Program (Grant no. 10028438, D12081) funded by the Ministry of Trade, Industry \& Energy (MI, Korea).

\section{References}

[1] WTO Scientific Group on the Burden of Musculoskeletal Conditions at the Start of the New Millennium, "The burden of musculoskeletal conditions at the start of the new millennium," World Health Organization Technical Report Series 919, 2003.

[2] L. K. P. Suen, T. K. S. Wong, J. W. Y. Chung, and V. Y. B. Yip, "Auriculotherapy on low back pain in the elderly," Complementary Therapies in Clinical Practice, vol. 13, no. 1, pp. 63-69, 2007. 
[3] Y. S. He, "Relaxing needling in combination with paired needling for 50 cases of acute lumbar sprain: learning from patients and the courts," World Journal of AcupunctureMoxibustion, vol. 22, no. 3, pp. 47-51, 2012.

[4] P. Whittaker, "Laser acupuncture: past, present, and future," Lasers in Medical Science, vol. 19, no. 2, pp. 69-80, 2004.

[5] G. D. Baxter, C. Bleakley, and S. McDonough, "Clinical effectiveness of laser acupuncture: a systematic review," Journal of Acupuncture and Meridian Studies, vol. 1, no. 2, pp. 65-82, 2008.

[6] R. T. Chow and L. Barnsley, "Systematic review of the literature of low-level laser therapy (LLLT) in the management of neck pain," Lasers in Surgery and Medicine, vol. 37, no. 1, pp. 46-52, 2005.

[7] K. H. Beckmann, G. Meyer-Hamme, and S. Schröder, "Low level laser therapy for the treatment of diabetic foot ulcers: a critical survey," Evidence-Based Complementary and Alternative Medicine, vol. 2014, Article ID 626127, 9 pages, 2014.

[8] N. S. Ebneshahidi, M. Heshmatipour, A. Moghaddami, and P. Eghtesadi-Araghi, "The effects of laser acupuncture on chronic tension headache-a randomised controlled trial," Acupuncture in Medicine, vol. 23, no. 1, pp. 13-18, 2005.

[9] A. Gür, M. Karakoç, K. Nas, R. Çevik, J. Saraç, and E. Demir, "Efficacy of low power laser therapy in fibromyalgia: a singleblind, placebo-controlled trial," Lasers in Medical Science, vol. 17, no. 1, pp. 57-61, 2002.

[10] D. Irnich, N. Behrens, J. M. Gleditsch et al., "Immediate effects of dry needling and acupuncture at distant points in chronic neck pain: results of a randomized, double-blind, shamcontrolled crossover trial," Pain, vol. 99, no. 1-2, pp. 83-89, 2002.

[11] World Health Organization, WHO Draft Guidelines for Adverse Event Reporting and Learning Systems, World Health Organization, 2005.

[12] B. Spilker, Quality of Life and Pharmacoeconomics in Clinical Trials, Lippincott-Raven, Philadelphia, Pa, USA, 2nd edition, 1996.

[13] Y. Y. Tian, "Preliminary observation of different cuppingnegative pressures on skin blood flow," Acupuncture Research, vol. 32, no. 3, pp. 184-185, 2007.

[14] O. S. Kwon, S. H. Lee, S. M. Choi, and Y. H. Ryu, "A study of researchpatterns for standardization of cupping therapy," Korean Journal of Acupuncture, vol. 29, no. 2, pp. 250-259, 2012.

[15] Korean Acupuncture \& Moxibustion Society Textbook Compilation Committee, The Acupuncture and Moxibustion, Jipmoondang, Seoul, Republic of Korea, 1988.

[16] R. T. Chow, M. I. Johnson, R. A. Lopes-Martins, and J. M. Bjordal, "Efficacy of low-level laser therapy in the management of neck pain: a systematicreview and meta-analysis ofrandomised placebo or active-treatment controlled trials," The Lancet, vol. 374, no. 9705, pp. 1897-1908, 2009.

[17] Y. B. Chae, Y. J. Kim, I. H. Choe, S. Lim, S. J. Lee, and H. J. Lee, “A comparison study of acupuncture sensation scale between real acupuncture and sham needle," The Korean Journal of Meridian \& Acupoint, vol. 23, no. 4, pp. 85-99, 2006.

[18] F. Beissner and I. Marzolff, "Investigation of acupuncture sensation patterns under sensory deprivation using a geographic information system," Evidence-Based Complementary and Alternative Medicine, vol. 2012, Article ID 591304, 10 pages, 2012. 


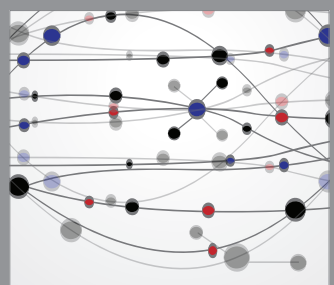

The Scientific World Journal
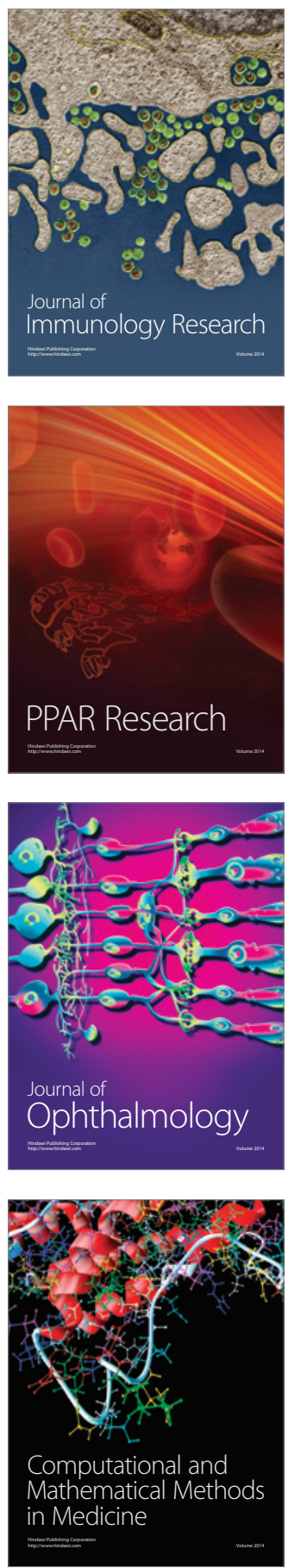

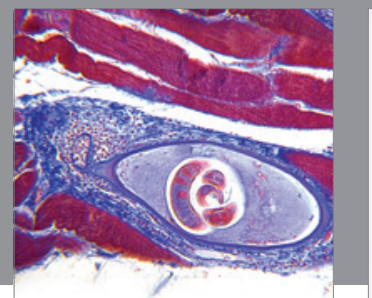

Gastroenterology

Research and Practice
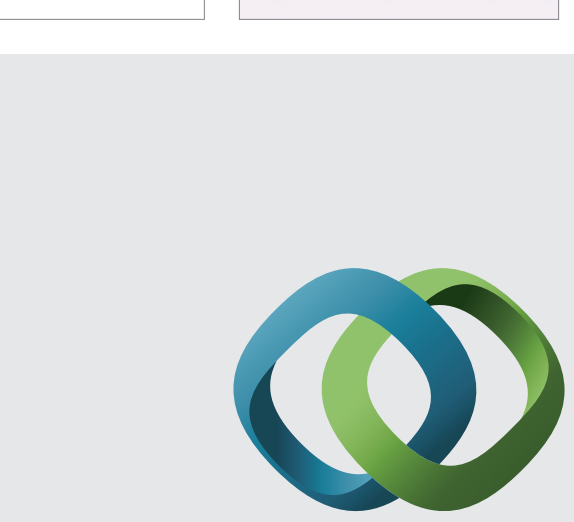

\section{Hindawi}

Submit your manuscripts at

http://www.hindawi.com
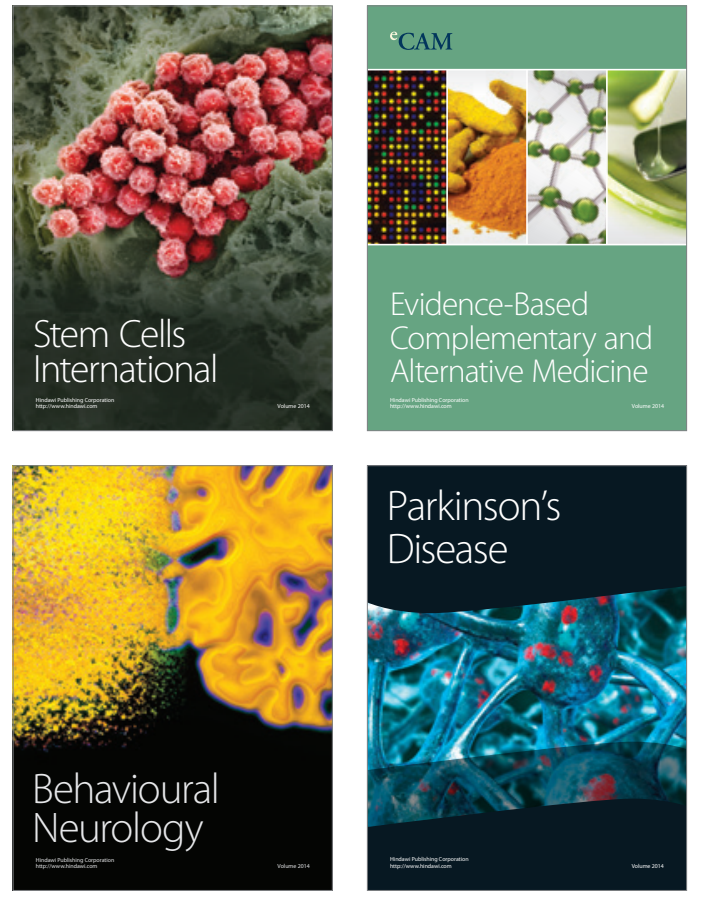
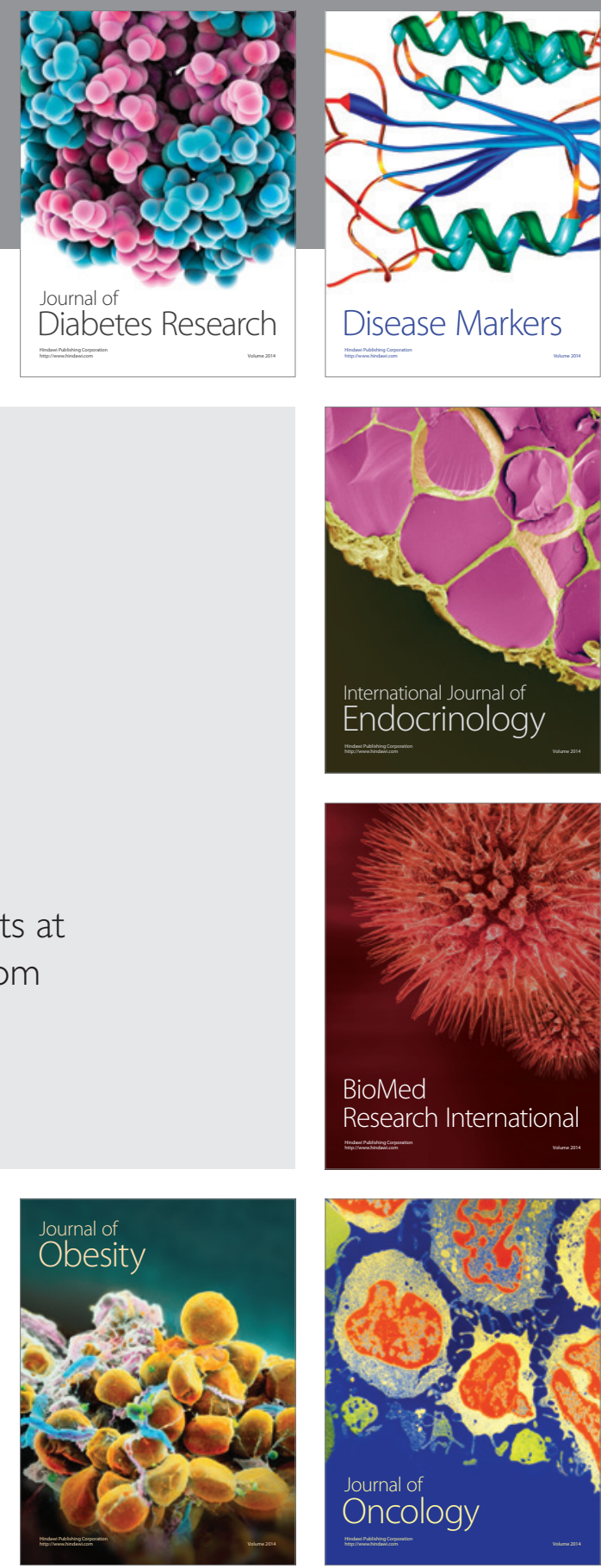

Disease Markers
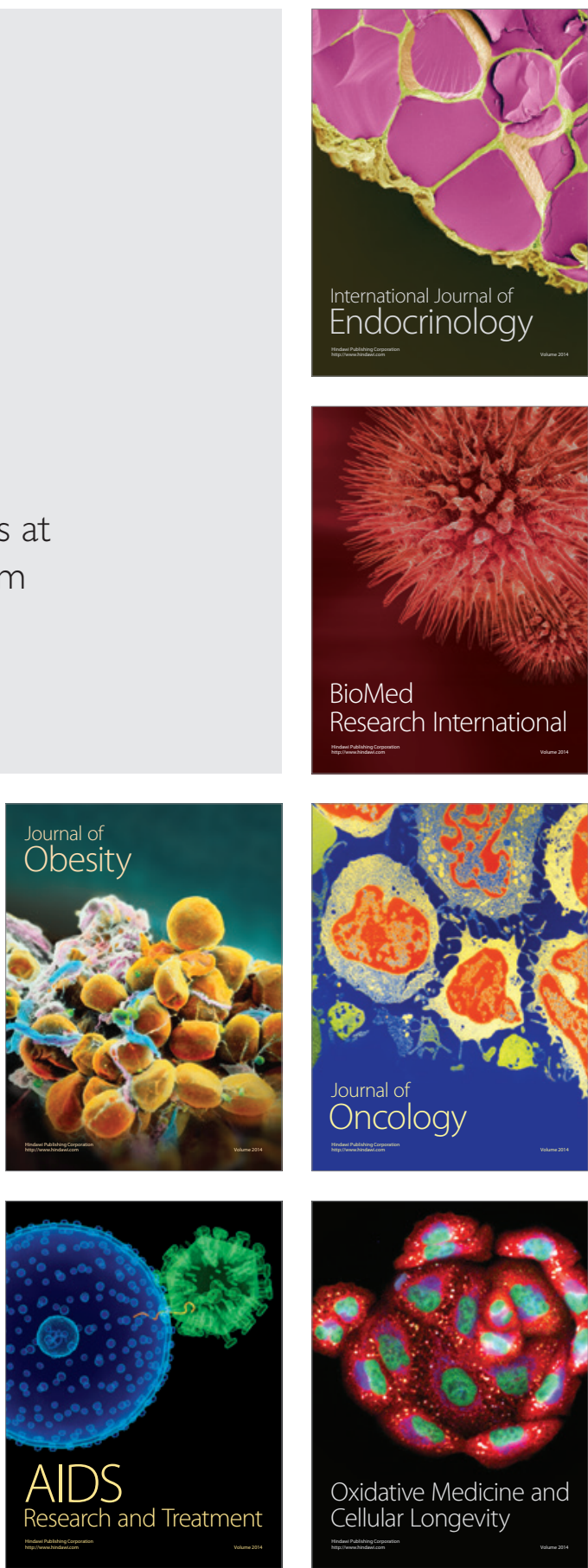